\title{
Warum pendeln manche Migranten häufig zwischen Herkunfts- und Ankunftsregion?
}

\author{
Eine empirische Untersuchung transnationaler Migration zwischen Mexiko und \\ den USA
}

\section{Von Ludger Pries}

Zusammenfassung: Auf der Basis der Ausbildungs-, Wohn- und Erwerbsverläufe von 648 mexikanischen Arbeitsmigranten, die in New York City zumindest eine Beschäftigung angaben, sowie der Auswertung qualitativer Feldstudien und biographischer Interviews zeigen sich bei einem Teil der Befragten als ,transnational' zu bezeichnende Migrationsmuster. Diese transnationalen Arbeitsmigranten pendeln wiederholt zwischen beiden Ländern, wobei es sich jeweils um mehrjährige Arbeitsaufenthalte in den USA handelt. Diese Migranten sind keine, gescheiterten' Einwanderer oder Rückkehrer, ihre Migrationsverläufe sind vielmehr Teil dichter transnationaler Familiennetzwerke. Die Auswertung der Lebensverläufe konzentriert sich auf die Erklärung der Anzahl der Landeswechsel durch personen-, familien-, zeit- und arbeitsbezogene Variablen. Die Befunde sind für das Verständnis von internationalen und hier besonders: von transnationalen Migrations- und Inkorporationsprozessen sowie für die gesellschaftliche Gestaltung einer angemessenen Anerkennung und Integration von transnationalen Migranten von großer Bedeutung.

\section{Einleitung}

Seit den 1990er Jahren haben sich Studien zu Transnationalisierungsprozessen im Allgemeinen und zu transnationaler Migration im Besonderen zu einem wichtigen interdisziplinären Forschungsfeld entwickelt. Als Stärke dieser Untersuchungsperspektive wird dabei angesehen, dass Verflechtungsbeziehungen ins Zentrum der wissenschaftlichen Analyse gestellt werden, die nationalstaatliche Grenzen überschreiten und relativ dauerhaft und verdichtet sind. Nach Alejandro Portes et al. besteht dabei Konsens über das vor allem interessierende Phänomen, nämlich "a growing number of persons who live dual lives: speaking two languages, having homes in two countries, and making a living through continuous regular contact across national borders" (Portes et al. 1999: 217). Unstrittig ist auch, dass transnationale Migration durchaus kein völlig neues Phänomen ist, wohl aber die entsprechende Forschungsperspektive innovativ sei. Schließlich besteht in der Forschung weitgehend Konsens darüber, dass transnationale Migranten zwar eine quantitativ vergleichsweise kleine Gruppe innerhalb aller internationalen Migranten ausmachen, dass die ökonomischen, politischen, sozialen und kulturellen Wirkungen der ,Transmigration' jedoch wesentlich größer sind, als ihr rein zahlenmäßiges Gewicht zunächst vermuten lässt (Portes 2003; Faist 2000 a und b; Pries 2005 a).

Die Forschung zu transnationaler Migration wird bisher größtenteils von qualitativen Feldund Fallstudien dominiert, die sich in den 1990er Jahren zunächst darauf konzentrierten, die Existenz transnationaler Migrationswirklichkeiten nachzuweisen (Kearney / Nagengast 1989; Smith 1997 und 2005; Goldring 1996, 1997 und 2001; Portes 1996; Basch et al. 1997; Weiß 2005; Lucassen 2006). Sowohl hinsichtlich des tatsächlichen Verbreitungsgrades von transnationalen Migranten als auch der Faktoren, die das Entstehen und die Stabilität transnationaler Migration beeinflussen, besteht ein großer Bedarf an repräsentativen oder zumindest mit größeren Fallzahlen operierenden Studien. So schlugen Portes et al. (1999) vor, sich in der weiteren Forschung auf drei Aufgaben zu konzentrieren: (1) die quantitative Verbreitung von Transmigranten in gegebenen Migrantenpopulationen genauer zu erfassen, (2) nur solche Phänomene als transnationale Migration zu benennen, die nicht durch andere, bereits bewährte 
Konzepte erklärt werden können und (3) die Dauerhaftigkeit transnationaler Phänomene besonders zu betrachten.

Die folgenden Ausführungen knüpfen an dieses Forschungsprogramm an und beschäftigen sich mit transnationaler Migration zwischen Mexiko und den USA. Hierzu liegt bereits eine Reihe von soziologischen und anthropologischen Fallstudien vor (Wiest 1973; Smith 2005; Goldring 1996 und 1997; Herrera Lima / Macías Gamboa 1998; Cohen 2001; Herrera Lima 2002). Massey et al. (1991) haben umfangreiche quantitative Erhebungen zu den Migrationsverläufen auf der Basis von Gemeindestudien vorgelegt und Espinoza / Massey (1997) wiesen empirische Evidenz für die Wirksamkeit des 'sozialen Kapitals' nach, das aus transnationalen sozialen Netzwerkverbindungen der Migranten erwächst. In neueren Untersuchungen haben Portes et. al (2002) und Portes (2003) transnationale mexikanische Kleinunternehmer untersucht. Diese Studien betrachten transnationale Migrationsströme hauptsächlich auf der Gemeinde- oder Länderebene. Demgegenüber werden im Folgenden vornehmlich transnationale Familien, Haushalte und Netzwerke betrachtet. Diese Perspektive knüpft an Arbeiten z.B. von Bryceson / Vuorela (2002), Faulstich et al. (2001) und Guarnizo et al. (2003) an. Guarnizo et al. (2003) plädierten dafür, die wirtschaftlichen und die nicht-ökonomischen Transaktionen der Migranten gemeinsam zu betrachten. Cohen (2001) und Conway / Cohen (2003) haben schwerpunktmäßig die auf Migration und Geldrücküberweisungen bezogenen Entscheidungsprozesse in mexikanischen Haushalten untersucht und konnten bei ihren qualitativen Fallstudien zeigen, dass die Entscheidungen und Strategien der einzelnen Migranten fast immer im Rahmen von komplexeren Haushalten und Großfamilien getroffen werden. Im Folgenden werden Ergebnisse einer empirischen Studie über Arbeitsmigranten zwischen Puebla/Mexiko und New York City vorgestellt, die diese Ergebnisse bestätigen, aber auch differenzieren. Vor allem stellt sich die Frage, warum ein Teil der befragten Migranten zwischen den beiden Ländern Mexiko und den USA den Wohnsitz und den Arbeitsplatz so häufig wechselt. Wieso pendeln Migranten Tausende von Kilometern zwischen Mexiko und den USA hin und her? Haben sie etwa als ,normale Einwanderer', die sich dauerhaft in den USA niederlassen wollen, versagt? Warum gehen sie dann nicht dauerhaft als Rückkehrmigranten in ihre Heimatregion in Mexiko zurück?

Um die vielen Grenzübergänge einer beträchtlichen Anzahl von Arbeitsmigranten - die weder grenznahe Pendler noch Saisonarbeiter sind - zwischen Mexiko und den USA zu erklären, wird im Folgenden das Argument entwickelt, dass solche Migrationsphänomene am besten durch das Konzept der transnationalen Migration bzw. der Transmigration erklärt werden können. In Abschnitt 2 wird das Design einer Untersuchung dargestellt, in deren Rahmen Pendelmigranten zwischen Mexiko und den USA befragt wurden. Dabei werden die generellen methodischen Probleme und Herausforderungen einer repräsentativen Untersuchung zu Transmigration diskutiert, und es wird ein methodischer Lösungsvorschlag vorgestellt. Abschnitt 3 präsentiert die Ergebnisse der Untersuchung. Sie verweisen auf einen bisher nicht explizit beachteten Typus von Migranten, der mit den herkömmlichen Kategorisierungen der Migrationsforschung nicht adäquat erfasst werden kann. Ein zentrales Merkmal dieses Typus des Transmigranten ist, dass er mehrfache grenzüberschreitende Wanderungen auch über sehr weite Entfernungen und für größere Aufenthaltszeiträume unternimmt. In Abschnitt 4 wird dieser genuin transnationale Aspekt näher untersucht. Im Mittelpunkt steht dabei, die Anzahl der grenzüberschreitenden Arbeits- und Wohnaufenthalte der befragten Migranten mithilfe von relevanten personen-, zeit-, gemeinde- und arbeitsbezogenen Merkmalen zu erklären. Im letzten Abschnitt wird dann die Transmigrationshypothese auf die Forschungsergebnisse angewendet, und es werden Vorschläge für die weitere Forschung gemacht (Abschnitt 5). 


\section{Daten und Forschungsdesign}

Forschungen über internationale Migration basieren in der Regel entweder auf sekundärstatistischen Analysen repräsentativer Daten aus Haushalts-, allgemeinen Bevölkerungs- und Arbeitsmarktumfragen oder auf (nicht repräsentativen) qualitativen Fallstudien in ausgewählten Bereichen. Während dem ersten Typus von Daten häufig kontextuelle Hintergrundinformationen und angemessene Variablen für spezifische Forschungsfragen fehlen, ist der zweite Typus von Daten bezüglich der Verallgemeinerbarkeit seiner Befunde und in der Durchführung von statistischen Analysen beschränkt. Für das Forschungsprojekt „Internationale Arbeitsmigration und die Herausbildung Transnationaler Sozialräume: das Fallbeispiel der Migration zwischen Puebla/Mexiko und dem Großraum New York" wurde auf der Grundlage von Ergebnissen qualitativer Studien (Kearney / Nagengast 1989; Smith 2005; Goldring 1996) die Hypothese sich herausbildender transnationaler Sozialräume zugrundegelegt. Häufige (und nicht nur saisonale) Landeswechsel in der Erwerbskarriere sind demnach nicht unbedingt Ausdruck von gescheiterten Auswanderungs- bzw. Rückkehrwanderungsstrategien, sondern von transnationalen Arbeits- und Lebensstrategien. ${ }^{1}$

Die Entscheidung, Arbeitsmigration von Puebla nach New York zu untersuchen, wurde auf der Basis verschiedener Kriterien getroffen. Vorausgehende Forschungen zeigten eine lange Tradition der Arbeitsmigration aus der Mixteca-Region (welche Teile der mexikanischen Bundesstaaten Puebla, Guerrero und Oaxaca umfasst) in den Nordwesten Mexikos und an die Westküste der USA (hauptsächlich Kalifornien; vgl. Chimal 1990; Hernández 1990; Motta 1990; Velasco Ortiz 1995). Es gab eindeutige Hinweise auf die wachsende Bedeutung der Geldrücküberweisungen für die lokale Wirtschaft in der Mixteca-Region (López / Cederström 1990; Cederström 1993; Cortés 1995 a, 1995 b und 1996). Weitere Studien stellten zunehmende Migrationsbewegungen aus dem Bundesstaat Puebla in die Region New York fest (Preibisch 1996; Valdéz 1994 und 1996; Smith 2005).

Im Vergleich zu den Forschungen über die nordwestlichen mexikanischen Bundesstaaten wie Jalisco und Michoacan (López Castro 1986 und 1988; Mummert 1990; Massey et al. 1991; Durand/Massey 1992; González et al. 1995) existierten bis in die 1990er Jahre nur wenige Studien über die Migrationsbewegungen aus den südöstlichen mexikanischen Bundesstaaten in die USA. Viele Indizien sprechen dafür, dass die grenzüberschreitenden Migrationsbewegungen aus dieser Region qualitativ und quantitativ an Bedeutung gewonnen haben. Bezüglich des quantitativen Ausmaßes nimmt Corona (1993) an, dass der Anteil der Migranten aus dem Bundesstaat Puebla an der gesamten mexikanischen Migrationspopulation in die USA im Zeitraum von 1960 bis 1990 von 2,8 \% auf 12, 5 \% anstieg (Herrera Lima / Macias Gamboa 1998). Parallel dazu verweisen Studien auf qualitativ neue Formen der Migration im Rahmen transnationaler Gemeinden (,transnational communities': Smith 2005), auf eine transnationale Musikkultur ${ }^{2}$ sowie auf komplexe und dynamische Familiennetzwerke, die verschiedene Orte in Mexiko und in den USA miteinander verbinden (Herrera Lima 2001; Cohen 2001).

Vor dem Hintergrund dieser Forschungslage wurde die Studie als ein Mix aus quantitativer und qualitativer soziologisch-anthropologischer Forschung konzeptionalisiert. Dazu wurden

1 Das Projekt wurde zwischen 1995 und 2001 durchgeführt und vom Mexikanischen Rat für Wissenschaft und Technologie (CONACYT) sowie von der Autonomen Metropolitanen Universität in Mexiko Stadt (UAM-Iztapalapa) finanziell unterstützt. Eine Beschreibung des Forschungsdesigns und einige vorläufige Befunde finden sich auch unter Macias Gamboa / Herrera Lima (1997).

2 Im Jahre 1995 sendete das lokale Radio FM 105.3 in Puebla Programmreihen mit Liedern, die von mexikanischen Migranten in New York komponiert und geschrieben wurden und in New York von den mexikanischen Musikern Enriqueta Silva, Òscar López und Carlos Arellano ausgearbeitet wurden. Auf diese Weise entstand eine Musik, die weder US-amerikanisch noch mexikanisch, sondern transnational war. 
fünf aus jeweils mehreren zusammenhängenden Gemeinden bestehende Herkunftsmikroregionen von Migranten definiert, die hinsichtlich ihrer jeweiligen sozio-ökonomischen Entwicklung, der Kommunikations- und Transportinfrastruktur sowie der Migrationsgeschichte starke Unterschiede aufwiesen: Chinantla-Piaxtla (ländliches Gebiet mit langer Migrationstradition), Acatlán/Huajuapan/Tehuitzingo (ländliches Gebiet, neu einsetzende Migrationsströme), suburbane Zonen von Atlixco (ländliche und städtische Gebiete, neu einsetzende Migrationsströme), Atlixco-Stadt (städtisches Gebiet, nachlassende Textilindustrie und neuere Migration) und die Industriegürtel zwischen den Städten Tlaxcala und Puebla (städtisches Gebiet, moderne Industriestruktur und neue Migrationsbewegungen). ${ }^{3}$ Der Großraum New York City (inklusive der benachbarten Gebiete im Bundesstaat New York und New Jersey) wurde als Ankunftsregion definiert.

Es wurden drei verschiedene Methoden der Datengewinnung angewandt. Erstens wurden lebensverlaufsbezogene, ereignisorientierte Datensätze durch persönliche Interviews mit Migranten erhoben (Themenfelder Herkunfts-, eigene und Nachkommengeneration, Bildungs-, Wohn- und Arbeitsverlauf); zweitens wurden autobiographische Lebens- und Migrationserzählungen von Migranten in persönlichen Interviews aufgenommen und transkribiert $(n=40)$; schließlich wurden drittens in der Herkunfts- und in der Ankunftsregion ethnographische Feldforschungsaufenthalte organisiert und systematisch ausgewertet. Da im Folgenden vorwiegend die Ergebnisse der Lebensverlaufserhebung vorgestellt werden, seien hierzu einige zusätzliche Informationen gegeben (vgl. Macías Gamboa / Herrera Lima 1998). Befragt wurden zwischen 1996 und 1998 Mexikaner, die die folgenden drei Bedingungen erfüllten: erstens mindestens 18 Jahre zu sein, zweitens aus einer der fünf Herkunftsmikroregionen zu stammen und drittens mindestens einmal im Leben zur Arbeit in die Großregion New York migriert zu sein. Da das Ziel darin bestand, eine möglichst gleiche Anzahl von Personen aus den fünf Entsenderegionen zu erhalten, wurden die Interviewpartner in einem doppelten Verfahren aus systematischem Zufallsauswählen nach Straßenzügen in den definierten Gemeinden (random walking) einerseits sowie daran anschließendem Vermitteln weiterer Interviewpartner (Schneeballsystem) ausgewählt.

Der Erhebungsbogen war in zwei Teile unterteilt. Ein Teil bezog sich auf persönliche Merkmale des Interviewten, seiner Eltern, Partner und (möglichen) Kinder; ein zweiter Teil bezog sich ereignisorientiert auf alle Arbeitsereignisse im Erwerbsleben des Interviewten. Auf diesem Wege wurden die individuelle und familiäre Situation sowie die Geschichte jedes Interviewten mit 150 personenbezogenen Variablen aufgenommen. Diese beinhalteten unter anderem: alle Migrationsbewegungen der Eltern, Geschwister und Kinder; Arbeit und Ausbildung der Eltern und des Partners; Ausbildung der interviewten Person, Trennung von den Eltern, Struktur des Haushaltes, Aufenthaltsorte im Lebensverlauf, Verwandtschaftsbeziehungen, Freundschaftsbeziehungen etc. Der Inhalt des verlaufsorientierten Arbeits- und Migrationsteils erhob jeweils 24 auf das Berufsleben bezogene Variablen für jedes aufgenommene Beschäftigungsverhältnis. Hier wurde unter anderem nach der Dauer der Beschäftigung, nach Ort, Art und Branche der Beschäftigung, nach Nationalität des Arbeitgebers, nach Arbeitszeiten, nach Art und Weise der Informationsbeschaffung bei der Jobsuche, nach den Adressaten von Geldrücküberweisungen, nach dem Verdienstniveau im Vergleich zur vorherigen Arbeitsstelle sowie nach den Gründen für den Wechsel der Arbeitsstelle gefragt.

3 Eine dieser beiden zuletzt einbezogenen Mikro-Regionen befindet sich im Grenzbereich zwischen den beiden mexikanischen Bundesstaaten Tlaxcala und Puebla. Um die Darstellung der geographischen Bezüge nicht unnötig kompliziert zu gestalten, wird hier im Weiteren nur von Puebla/Mexiko als der Herkunftsgroßregion gesprochen. 
Insgesamt führte das Forschungsteam etwa 800 ereignisorientierte Interviews durch, welche dann kontrolliert und analysiert wurden. ${ }^{4}$ In die statistische Analyse gingen 648 gültige Fragebögen in kodifizierter Form auf der Basis von zwei unterschiedlichen SPSS-Dateien (eine personen- und eine berufsverlaufsbezogene) ein. Die untersuchte Stichprobe bestand aus 200 Frauen und 448 Männern, deren Durchschnittsalter bei 32,3 Jahren lag. Aus den fünf Herkunftsmikroregionen wurden jeweils zwischen 105 und 161 Personen interviewt. Mit einem Gesamtwert von 3060 gültigen Ereignissen entfallen auf jede interviewte Person im Durchschnitt 4,7 Beschäftigungsereignisse. Im Durchschnitt dauerten die Beschäftigungsverhältnisse 3,6 Jahre. Jede Person pendelte durchschnittlich 2,4 Mal zwischen Mexiko und den USA; 126 Personen unternahmen mindestens vier Arbeitswanderungen zwischen den beiden Ländern.

Strukturelle Probleme machen verlässliche Aussagen über die Repräsentativität der Befragungsergebnisse unmöglich. Ein erstes grundlegendes Defizit besteht darin, dass über die Grundgesamtheit, über die Aussagen gemacht werden sollen - die Gesamtheit der zwischen den definierten Herkunfts- und Ankunftsregionen migrierenden Mexikaner - keinerlei ,harte' Informationen vorliegen. Da der überwiegende Teil der betreffenden Personen als indocumentados, also ohne legale Erlaubnis die Landeswechsel und Jobaufnahme organisiert (bei den befragten 648 Migranten fast neun Zehntel aller Jobereignisse) und weder in den Herkunfts- noch in den Ankunftsregionen irgend eine Art von Register über die Migrierenden geführt wird, wäre nur durch eine Vollerhebung der gesamten Bevölkerung in den entsprechenden Regionen eine Definition der Grundgesamtheit aller Migrierenden möglich. Die Unsicherheit über die Verhältnisse in der Grundgesamtheit zeigt sich z.B. in stark divergenten Schätzungen: Für die Mitte der 1990er Jahre gab das mexikanische Nationale Institut für Statistik, Geographie und Informatik (INEGI) die Anzahl der Migranten aus Puebla in die USA insgesamt mit nur etwa 47.000 Personen an. Für die gleiche Zeit wurde die Anzahl aller Mexikaner allein im Großraum New York (in dem die Migranten aus dem Bundesstaat Puebla die Mehrheit bilden sollen) auf 150.000 bis 350.000 geschätzt (Valdéz 1994; Smith 2005).

Ein zweites Problem bezüglich der Repräsentativität ergibt sich aus der dynamischen und informellen Situation der Migranten und ihrer Familien in verschiedenen Bereichen: Was ist ein angemessener Referenzrahmen für die Auswahl der zu interviewenden Personen? Bei Arbeitsmigranten könnte man theoretisch die Personen in der Ankunftsregion auf der Basis von Arbeitseinheiten, in denen sie beschäftigt sind, aussuchen. Jedoch ist es fast unmöglich, einen Zugang zu den Arbeitsplätzen der Migranten zu bekommen (illegale sweat shops, Arbeit in privaten Haushalten, Arbeit als Gärtner oder private Kellnerin). Außerdem hätte man über diese Methode nur Zugang zu Migranten, die gerade aktiv in der Ankunftsregion arbeiten. Zurückgekehrte Migranten wären somit systematisch ausgeschlossen.

Eine zweite Möglichkeit bestünde darin, in ausgewählten Mikroregionen alle Haushalte zu erheben und z.B. den Haushaltsvorstand als Referenzpunkt für die Auswahl von Interviewkandidaten zu befragen. ${ }^{5}$ Leider zeigte sich in der Erhebung, dass die Haushaltszusammensetzung der betreffenden Migranten sehr dynamisch ist: Nach welchem Kriterium auch immer der Haushaltsvorstand bestimmt wurde (die Person, die das meiste Geld einbringt oder die Person, die die meiste Zeit arbeitet): In nur wenigen Jahren änderte sich - z.B. durch Migra-

4 Neben den direkten Forschungsteammitgliedern wurden 24 Interviewer geschult und eingesetzt; häufig handelte es sich dabei um qualifizierte Personen aus der Region als, gate keeper', z.B. Dorflehrer, Ärzte und Studenten.

5 Das inzwischen an der University of Princeton angesiedelte Mexican Migration Project hat auf der Basis dieses Prinzips Daten in mehr als 100 mexikanischen Gemeinden erhoben. Diese Studie beinhaltet inzwischen auch mehrere Gemeinden in den Bundesstaaten Oaxaca und Puebla; zum Zeitpunkt unserer eigenen Studie waren allerdings noch keine Daten für die von uns untersuchten Gemeinden ausgewertet (vgl. http://mmp.opr.princeton.edu/). 
tion - sowohl die Gesamtzusammensetzung der Haushaltsmitglieder als auch der jeweilige Haushaltsvorstand. Deshalb tauchen auch bei dem möglichen Vorgehen, über eine Vorbefragung von Haushaltsvorständen ein verlässliches Kataster aller Migrierenden zu erhalten, erhebliche Schwierigkeiten auf. So ist z.B. die Definition des Haushaltsvorstandes nach dem Kriterium des größten Beitrags zur Haushaltsökonomie durchaus fragwürdig. Ist z.B. ein in einer mexikanischen Gemeinde als Haushaltsvorstand Registrierter auch später noch als solcher zu verstehen und zu führen, wenn er in den USA als Migrant arbeitet (und real vielleicht dann noch mehr Geld zum Haushalt in Mexiko beisteuert als vorher). Wie soll man pluri-lokale transnationale Haushalte berücksichtigen? Von den 648 interviewten Personen gaben 377 an, zum Interviewzeitpunkt den Hauptverdienst zu ihrem jeweiligen Haushalt beizutragen; 163 trugen demnach den zweitgrößten Verdienst bei (beide Gruppen machen zusammen 83 Prozent der Interviewten aus). Häufig wechselte die Position des Hauptverdieners eines Haushaltes innerhalb weniger Monate eines Jahres.

Aufgrund der beschriebenen Schwierigkeiten, eine exakte Bestimmung der Grundgesamtheit vorzunehmen, aus der eine repräsentative Zufallsstichprobe gezogen werden könnte, wurde mit der durchgeführten Untersuchung keine Repräsentativität angestrebt, wohl aber eine methodisch kontrollierte und bestimmte Bedingungsfaktoren kontrollierende Auswahl der Interviewpartner als Erhebungseinheiten. Dabei wurden drei Prinzipien miteinander verbunden: Die fünf Mikroregionen im Herkunftsland Mexiko wurden nach dem Prinzip maximaler Kontraste und des theoretical sampling ausgewählt; innerhalb dieser Mikroregionen wurden nach dem Zufallsprinzip jedes dritte Haus in festgelegten Straßenzügen (random walking) für Haushaltsbefragungen ausgewählt, und schließlich wurden in einer weiteren Erhebungswelle nach dem Schneeballprinzip neue Interviewpartner gewonnen. Um die Informationen über einzelne Haushalte nicht zu verdoppeln, wurde immer nur jeweils ein Mitglied eines Haushaltes ausgewählt. Insgesamt 57 Befragungen wurden in der Großregion von New York City nach dem Schneeballprinzip durchgeführt.

\section{Transmigration als neues Muster der Migration}

Eine erste Analyse, die sich auf die erwerbsverlaufsorientierten Daten konzentrierte, ergab deutliche Hinweise auf neue Migrationsphänomene und auf die Emergenz von Transmigranten als einem neuen Typus internationaler Migranten. Die klassischen Arbeitsmigranten, die von Mexiko in die USA wanderten, waren Personen mit geringem Bildungshintergrund aus ländlichen Gebieten, die vor allem aus dem Nord-Westen Mexikos in vorwiegend landwirtschaftliche und manuell-industrielle Beschäftigungssektoren im Westen und Süden der USA wanderten. Laut Bustamante / Martinez (1979: 268) stammten Ende der 1970er Jahre 80 \% aller mexikanischen Migranten aus den folgenden Bundesstaaten: Baja California Norte, Chihuahua, Durango, Guanajuato, Guerrero, Jalisco, Michoacán, San Luis Potosí, Sinaloa Zacatecas. Laut einer Bevölkerungsumfrage in den USA aus dem Jahre 1980 lebten $87 \%$ der legalen und $84 \%$ der undokumentierten mexikanischen Migranten in den folgenden US-Staaten: Arizona, California, Colorado, New Mexiko und Texas (Heer 1993: 78ff). Dies zeigt, dass die Mexiko-USA-Migration traditionell aus bestimmten Herkunftsregionen und in spezifische Ankunftsregionen stattfand.

Verglichen mit diesen klassischen Migrationsströmen zeigen sich bei der Untersuchung der Migrationsbewegungen zwischen Puebla und New York neue Muster. Von den 648 Interviewten gaben immerhin 180 an, ihre erste Beschäftigung überhaupt auf dem US-amerikanischen Arbeitsmarkt aufgenommen zu haben, und zwar größtenteils direkt im Großraum New York. Dies bedeutet, dass sie nicht an Kettenmigrationsbewegungen - z.B. zunächst entlang der traditionellen Route in die Südstaaten der USA und dann von dort weiter in Richtung NordOsten der USA - teilnahmen. Die Datenanalyse nach Kohorten des ersten US-amerikanischen 
Jobs zeigt, dass eine hohe Anzahl von Migranten mit einem hohen Bildungsstand ihre erste Beschäftigung im Dienstleistungsbereich, meist in kleineren Unternehmen mit bis zu 20 Beschäftigten, aufnimmt. Im Großraum New York City angekommen, bewegen sich die mexikanischen Migranten in einer segmentierten Arbeitswelt aus Mexikanern und anderen Lateinamerikanern (weniger als $5 \%$ aller Interviewten gaben an, dass die Mehrzahl ihrer Kollegen weiße oder schwarze US-amerikanische Bürger seien).

Wenn man die mehrere Tausend Kilometer langen Distanzen zwischen dem Herkunfts- und dem Ankunftsgebiet berücksichtigt, stellt sich die Frage, wie die Wanderung selbst und z.B. der Grenzübergang organisiert werden. Soziale Netzwerke spielen hier eine entscheidende Rolle. Die Migranten reisen, organisieren ihre Unterkunft und suchen Arbeit im Kreis eines dichten Netzwerkes von Familienangehörigen, Freunden und weiteren Personen ihres Vertrauens (z.B. solchen aus der gleichen Gemeinde oder Region, Landsleuten, latinos etc.). Sie schicken Geld, um für den Lebensunterhalt ihrer Eltern, Partner und Kinder zu sorgen. Eine genauere Analyse des Eintritts in die und des Austritts aus der erste(n) Arbeitsstelle zeigt, dass die Migranten vorrangig mittels ihrer sozialen Netzwerke Informationen über Arbeitsgelegenheiten erhalten, eventuell notwendige Empfehlungen für den Beschäftiger besorgen sowie Tipps und Einweisungen über das angemessene Verhalten in der Arbeit bekommen. Auch die Suche nach relevanten Informationen zur Unterkunft, das Ausgeben des verdienten Geldes und die Geldüberweisungen finden in einem pluri-lokal und transnational aufgespannten sozialen Netzwerk statt. Schließlich kündigt die große Mehrheit der Befragten ihr erstes Arbeitsverhältnis in den USA , freiwillig' und aus privat-familiären Gründen (genannte Gründe: Heimweh, notwendige Hilfe bei Pflege der Eltern oder Kinder, Übernahme anderer wichtiger Funktionen im Familienkontext etc.). Eine bedeutende Anzahl von Migranten (mehr als ein Fünftel aller Befragten) hatten die US-mexikanische Grenze zum Befragungszeitpunkt mindestens viermal überquert. Der Migration aus Mexiko in die USA liegen ökonomische Gründe zugrunde, die Rückkehrwanderung aus den USA nach Mexiko erfolgt aus privat-familiären Gründen. Alle anderen (freiwilligen und unfreiwilligen) Erklärungsfaktoren für die Aufgabe der Beschäftigungsverhältnisse in den USA haben nur einen marginalen Erklärungswert.

Diese Befunde legen die Annahme nahe, dass neben den klassischen Idealtypen (1) des Auswanderers bzw. Einwanderers (der im Idealfall nur einmal die Grenze überschreitet und sich dann dauerhaft im Ankunftsland niederlässt), (2) des Rückkehrmigranten bzw. ,Gastarbeiters' (der im Idealfall die Grenze einmal zur Auswanderung und einmal zur Rückwanderung überschreitet und sich dann dauerhaft wieder im Herkunftsland niederlässt) und (3) des saisonalen Pendlers (der im Idealfall grenznah und sehr häufig für kurze jahreszeitbedingte Arbeitsaufenthalte hin- und herwandert) ein vierter Idealtyp internationaler Arbeitswanderung von Bedeutung ist, den man Transmigrant nennen kann. Dieser Typus des Transmigranten unterscheidet sich von den drei anderen, klassischen Typen durch seinen Wanderungsverlauf und seine Lebensorientierungen.

Dem Idealtypus des Einwanderers kommen diejenigen recht nahe, die in den Befragungen angaben, schon seit sehr vielen Jahren oder gar Jahrzehnten in den USA bzw. konkret im Großraum New York zu leben. Viele von ihnen schickten ihre Kinder auf Grund- und weiterführende Schulen, einige hatten bereits Wohneigentum erworben. In Gesprächen erläuterten sie, trotz aller Probleme ihren langfristigen Lebensmittelpunkt in den USA zu sehen. Eine andere Gruppe von Migranten hielt sich zum Befragungszeitpunkt wieder in Mexiko auf und gab an, auf der Suche nach Arbeit nicht bzw. niemals wieder in die USA zu wandern. Vorrangige Gründe hierfür waren negative Erfahrungen , auf der anderen Seite', langfristige familiäre Verpflichtungen und starke emotionale Bindungen an die Herkunftsregion oder auch die Tatsache, dass ihr Traum von einer kleinen selbständigen Existenz mithilfe des durch die 
Arbeitswanderung verdienten Geldes wahr geworden war. Diese Menschen kommen dem Idealtypus der Rückkehrmigranten sehr nahe.

Neben den ,Einwanderern' und den ,Rückkehrern' ließ sich aber auch eine dritte Gruppe von Interviewten identifizieren (je nach Enge oder Weite der Definition etwa ein Viertel bis ein Sechstel aller Befragten), die eine größere Anzahl an Hin- und Rückwanderungen zwischen Mexiko und den USA von jeweils ausgedehnten Zeiträumen mehrerer Jahre angab. Es handelte sich also nicht um saisonale Wanderer, von denen es in Mexiko sehr viele gibt und die vor allem zu den verschiedenen Ernteperioden in den Westen und Süden der USA migrieren. Diese Befragten waren auch keine grenznahen Pendelwanderer. Sie legten - fast ausschließlich als indocumentados, also Migranten ohne gültige Papiere - mehrere Tausend Kilometer zwischen ihrer Herkunftsregion und New York unter extremen Bedingungen zurück. Für die ersten ein oder zwei Grenzüberschreitungen hatten sie in der Regel einem ,pollero' (Schlepper) jeweils einige Hundert US-Dollar bezahlen müssen, die letzten Male hatten sie die Grenze nicht selten zusammen mit anderen erfahrenen Migranten allein überwunden. Diese Menschen benannten in Interviews Vorteile und Nachteile sowohl der Ankunfts- als auch der Herkunftsregion. Auf die Frage nach ihren langfristigen Lebensplänen gaben sie häufig an, nur für die nächsten zwei oder drei Jahre zu planen und dann zu sehen, was Gott mit mir vorhat'. Ein Teil ihrer Familie und ihres primären sozialen Netzes lebte in der Herkunftsregion in Mexiko, ein anderer Teil der ihnen wichtigen Menschen lebte in den USA. Diese Menschen kommen dem Idealtypus des Transmigranten sehr nahe.

Der typische Transmigrant unternahm fünf oder noch mehr Landeswechsel im Verlauf seiner Migrationsgeschichte und Erwerbsbiographie. ${ }^{6}$ Jedes Beschäftigungsverhältnis dauerte in der Regel mehrere Jahre. Die Gründe für die Beendigung eines Beschäftigungsverhältnisses (und für den damit verbundenen Landeswechsel) waren fast ausschließlich , freiwillig'. Unerwartet selten wurden ,Probleme mit dem Aufenthaltsstatus', ,persönliche Entlassungen“ oder , Geschäftsschließungen` als Gründe für die Beendigung einer Beschäftigung genannt - all diese nicht-freiwilligen Gründe machten insgesamt nur ungefähr ein Zehntel aller Landeswechsel aus. Dies ist vor allem vor dem Hintergrund der Tatsache bemerkenswert, dass die große Mehrheit aller Beschäftigungsverhältnisse (86\%) nach Auskunft der Befragten als indocumentados, also ohne formale Arbeitserlaubnis und ohne rechtliche Aufenthaltserlaubnis in den USA realisiert wurde. Diese vorwiegend freiwilligen Wechsel von Beschäftigung und Aufenthaltsland legen die Vermutung nahe, dass die Transmigranten nicht als , gescheiterte Einwanderer' bezeichnet werden können (Pries 1996 und 1998).

Die bisherigen Ausführungen legen eine Frage nahe: Warum nehmen so viele Befragte das hohe Risiko und die enormen Kosten von wiederholten Landeswechseln zwischen den USA und Mexiko auf sich? Aus den Lebenslaufbefragungen, den qualitativen biographischen Interviews und der ethnographischen Feldforschung ergibt sich als Antwort: Diese Menschen richten als Transmigranten ihre Erwerbsstrategien und häufig auch ihre familiären Lebensgewohnheiten pluri-lokal zwischen Mexiko und den USA ein. Die biographischen Lebensentwürfe und kollektiven Normalitätsstandards, das alltägliche Leben sowie der Lebensverlauf als eine Sequenz unterschiedlicher Beschäftigungen und Wohnsitze spannen sich in einem mehr oder weniger einheitlichen und dauerhaften Sozialraum über mehrere geographische Plätze hinweg auf. Durch dieses pluri-lokale und transnationale Leben können sie ihre Hand-

6 Mit Landeswechsel sind hier nur die im Zusammenhang eines Beschäftigungswechsels vorgenommenen Grenzüberschreitungen gemeint. Dies schließt nicht aus, dass ein Migrant z.B. während eines Beschäftigungsverhältnisses in den USA für eine kurze (Urlaubs- oder Feiertags-) Periode in seine mexikanische Heimatgemeinde fährt. In diesem Fall würde die Grenzüberschreitung, da nicht im Zusammenhang des Wechsels einer Beschäftigung erfolgt, nicht als ,Landeswechsel' gezählt. 
lungschancen erweitern, und in der Regel empfinden sie diese transnationalen Lebenswelten nicht nur als Herausforderung, sondern auch als Bereicherung.

Ein gutes Beispiel für die Existenz von transnationalen Sozialräumen und Biographien ist die Familie von Doña Rosa, aus der fünf verschiedene Personen unterschiedlicher Generationen interviewt wurden (Herrera Lima 2001). Doña Rosa war zum Interviewzeitpunkt über siebzig Jahre alt (an das genaue Geburtsjahr konnte sie selbst nicht erinnern). Ein Teil der Familie von Doña Rosa lebte in den USA (hauptsächlich im Großraum New York) und ein anderer Teil in Mexiko (größtenteils in der Mixteca-Poblana-Region). Viele Familienmitglieder unterschiedlicher Generationen wechselten häufiger ihren Wohnsitz zwischen diesen beiden Gebieten. Die zweitälteste Tochter Guadalupe war gerade dreizehn Jahre alt, als ein aus einem Nachbardorf stammender Arbeitsmigrant von einem Aufenthalt aus New York City zurückkehrte und nach einer Ehepartnerin Ausschau hielt. Guadalupe half ihrer Mutter beim Getränkeausschank, sie gefiel dem jungen Mann und dieser hielt um ihre Hand an. Doña Rosa ließ ihre Tochter Guadalupe nur unter der Bedingung heiraten und mitgehen, dass ihr ältester Sohn Antonio das neue Paar in die USA begleite. Auf diese Weise wurden bereits in den 1960er Jahren die ersten persönlichen und Arbeitsbeziehungen nach New York geknüpft. Als Guadalupe ihr zweites Kind gebar, kehrte sie zusammen mit ihrem Ehemann in ihr Heimatdorf zurück, beide wollten, dass ihre Kinder in Mexiko erzogen würden. Von dem ersparten Geld ihrer Arbeit in der Bekleidungsindustrie New Yorks eröffneten sie ein Einzelhandelsgeschäft.

Der älteste Sohn Doña Rosa's, Antonio, der seine Schwester Guadalupe nach New York begleitet hatte, arbeitete ebenfalls eine Zeit lang in einem, sweatshop' in New York, also einem kleinen Bekleidungsbetrieb, in dem T-Shirts oder Hosen zusammengenäht werden. Da er bereits sehr viele Erfahrungen in den unterschiedlichsten Tätigkeitsbereichen (Hühnerausnehmen auf dem Wochenmarkt, Assistent eines Verkaufsagenten, fahrender Verkäufer von Heiligenbildchen im Norden Mexikos etc.) gesammelt hatte, begann er in New York später eine Arbeit in einer Restaurant-Küche. Nachdem er hier viele Kenntnisse und etwas Geld erworben hatte, eröffnete er zusammen mit seiner Schwester Guadalupe in Yonkers (Bundesstaat New York) ein Spezialitätenrestaurant mit Angeboten aus der regionalen Küche der Mixteca-Poblana. Sonntags verkaufte Antonio in der Regel mexikanisches Essen an seine (vorwiegend ohne Aufenthaltspapiere in New York arbeitenden) Landsleute, die sich im Norden Manhattans am Hudson-River zum Fußballspielen trafen (Ende der 1990er Jahre gab es immerhin etwa 70 mexikanische Fußballmannschaften in New York, die in einer eigenen Liga um einen Jahrespokal spielten). Das von Guadalupe und Antonio gemeinsam in den 1970er Jahren aufgebaute Restaurant wurde auch Ende der 1990er Jahre noch von Antonio betrieben, und seine Schwester Guadalupe war weiterhin Mitbesitzerin. Obwohl sie inzwischen vorwiegend in Puebla/ Mexiko lebte, verbrachte sie jedes Jahr mehrere Monate bei ihrem Bruder in Yonkers. Guadalupes Mann wurde inzwischen im Heimatdorf als erster Rückkehrmigrant zum Gemeindepräsident gewählt und kümmerte sich vorwiegend um das dort eröffnete Geschäft.

Doña Rosa hat zwar ihren Lebensmittelpunkt inzwischen in der Mixteca-Poblana, aber ähnlich wie ihre Tochter Guadalupe fliegt sie regelmäßig nach New York, um den anderen Teil ihrer Familie zu besuchen. Wird irgendwo in der weitläufigen Nachkommenschaft ein Kind geboren, fährt sie für den notwendigen Zeitraum dorthin um zu helfen. Als ihre älteste Tochter Aurora in der mexikanischen Gemeinde mit finanzieller Unterstützung der Großfamilie und aus der Beobachtung heraus, dass gerade bei vielen in den USA lebenden mexikanischen Arbeitsmigranten speziell zu den Dorffesten eine große Nachfrage nach angemessenen Hotelunterkünften besteht, ein kleines Hotel aufbaute und mit dessen Führung einige Probleme hatte, kam Doña Rosa - damals gerade in New York lebend - nach Mexiko zurück, um bei der Stabilisierung des Hotelgeschäftes zu helfen. 
Neben dem Hotel betreibt Aurora noch ein Restaurant in der mexikanischen Gemeinde, welches sie von dem ersparten Geld aufbauen konnte, das sie bei ihrem anderen Bruder Ricardo in dessen kleinem Hotel in der Gemeinde verdient hatte. Der jüngste Sohn von Aurora lebt bei ihr in Mexiko; er ist in den USA geboren und besitzt also - wie auch viele andere der Nachkommenschaft von Doña Rosa - die US-amerikanische Staatsbürgerschaft. Dies gilt auch für ihre älteste Tochter, die inzwischen in New York mit einem mexikanischen Migranten verheiratet ist und schon selbst eine Tochter hat. Aurora selbst besitzt schon seit 1984 die USamerikanische Staatsbürgerschaft und kann deshalb - ebenso wie die meisten ihrer Großfamilie - ungehindert zwischen beiden Ländern reisen. Das bereits erwähnte kleine Hotel von Ricardo wird von einer zu Beginn der 1970er Jahre in New York City geborenen Tochter mexikanischer Migranten aus der Mixteca Poblana geleitet. Sie besitzt die US-amerikanische Staatsbürgerschaft, kam aber zum Studium nach Puebla, der Hauptstadt des gleichnamigen Bundesstaates zurück. Sie heiratete inzwischen einen mexikanischen Migranten in New York - wo sie zusammen mit ihrem Ehepartner langfristig leben wird - in den USA, in Mexiko oder transnational - kann sie gegenwärtig nach eigenem Bekunden noch nicht absehen.

Die komplexen Strukturen und die Geschichte der Großfamilie von Doña Rosa können hier nur angedeutet werden (vgl. Herrera Lima 2002). Die meisten Mitglieder der Familie Doña Rosa's sind hinsichtlich ihrer Berufskarrieren sicherlich überdurchschnittlich erfolgreich. Dennoch können die wenigen hier präsentierten Informationen einige für den Transnationalisierungszusammenhang bedeutsame Aspekte verdeutlichen. Internationale Arbeitsmigration erfolgt in der Regel als Kettenwanderung und im Rahmen sozialer Netzwerke. Erreicht internationale Migration eine bestimmte Größenordnung, so schafft sie eigene neue Nachfragestrukturen (z.B. nach mexikanischen Lebensmitteln und Restaurants in New York), die wiederum mehr Migration nach sich ziehen (zum Konzept der ,cumulative causation“ vgl. Massey et al. 1994 und 1998). Neue Nachfragestrukturen entstehen auch in den Herkunftsgemeinden der Migranten (z.B. nach kleinen Hotels für Auswanderer oder transnationale Migranten). Trotz - oder vielleicht gerade wegen - des Besitzes der US-amerikanischen Staatsbürgerschaft fühlt sich ein Teil der Arbeitsmigranten und ihrer Nachkommen für zumindest einen Teil ihres Lebens dem Herkunftsland der Vorfahren sehr verbunden. Gerade weil die doppelte Staatsbürgerschaft problemlos Hin- und Herbewegungen ermöglicht, können die Menschen je nach ihren sozialen, kulturellen, wirtschaftlichen und politischen Erfordernissen die ihnen bekannten und vertrauten Regionen in beiden Ländern als ihren natürlichen Aktionsraum begreifen und nutzen.

Wenn hier ein Teil der befragten mexikanischen Arbeitsmigranten als Transmigranten bezeichnet wird, so soll damit nicht der Eindruck erweckt werden, es handele sich überwiegend um souveräne neue ,Kosmopoliten', die sich völlig frei und freiwillig zwischen verschiedenen Orten, Regionen und angebotenen Möglichkeiten problemlos hin- und herbewegen. Es sollen keineswegs die häufig prekären Lebenssituationen von internationalen Migranten insgesamt und auch von vielen Transmigranten ausgeblendet werden. Transmigranten müssen sich an schwierige und nicht vorausschaubare Verhältnisse anpassen, sie lernen mit Risiken umzugehen und zu leben. Sie würden unter anderen Lebensumständen eventuell einfache Auswanderer oder Rückkehrwanderer sein. Ihre Lebensweise impliziert in vielfacher Hinsicht hohe Transaktionskosten. Was den Aufenthaltsort und die Arbeit betrifft, so ist ihre Lebensplanung nicht über einen längeren Zeitraum fixiert, sondern stärker sequentiell und darauf ausgerichtet, Möglichkeiten ad hoc auszunutzen und zu ergreifen.

Insofern sind Transmigranten nicht frei, die gesamten Umstände ihres Handelns selbst zu definieren. Aber diese Einschränkung gilt auch für diejenigen Migranten, die den anderen Idealtypen der Auswanderer und der Rückkehrwanderer nahe kommen. Die Transmigranten akkumulieren in der Regel stärker kulturelles und soziales Kapital (Espinoza / Massey 1997; 
Palloni et al. 2001). Da der Möglichkeitshorizont ihrer Entscheidungen und ihre Erwartungen weder nur auf die Herkunftsregion noch nur auf die Ankunftsregion beschränkt sind, eröffnen sich ihnen generell mehr Handlungsoptionen. Insofern ihre Familienbezüge in unterschiedlichen Gesellschaften dauerhaft und über Generationen verankert sind, kann eine transnationale Lebensstrategie bzw. eine Lebensstrategie in transnationalen Sozialräumen durchaus eine langfristige, individuell erfolgreiche und zufriedenstellende Lebensperspektive eröffnen. Transmigranten sollten also weder als Opfer gescheiterter klassischer Integration noch als völlig souveräne und von allen Handlungszwängen befreite neue ,Weltbürger' angesehen werden. Im folgenden Abschnitt wird spezifischer untersucht, welche personen-, familien-, zeit-, regionen- und beschäftigungsbezogenen Merkmale der befragten mexikanischen Arbeitsmigranten die Häufigkeit ihrer Beschäftigungs- und Landeswechsel erklären.

\section{Erklärungen für die Häufigkeit der Wanderungen zwischen Mexiko und den USA}

Angesichts der beobachteten, teilweise beträchtlichen Häufigkeit von Wanderungen zwischen Mexiko und den USA bei zumindest einem Teil der befragten mexikanischen Arbeitsmigranten ist diese Anzahl der Landeswechsel eine zentrale Variable, die einer näheren Analyse bedarf. Die Befragten gaben in der Untersuchung zwischen 2 und 14 arbeitsbedingte Landeswechsel zwischen Mexiko und den USA an. Die Auswertung der Daten zeigt, dass die Mexikaner aus ökonomischen Gründen in die USA migrieren und dass sie aus familiären Gründen nach Mexiko zurückkehren. Erklärungsbedürftig bleibt jedoch die unterschiedliche Häufigkeit der Wanderungen der Migranten. Welche Faktoren können zur Klärung der Frage beitragen, warum einige Migranten die Grenze bis zum Befragungszeitpunkt nur ein oder zwei Mal, andere dagegen zehn Mal oder noch häufiger überquert haben?

Eine mögliche Erklärung liegt in personenbezogenen Faktoren wie Geschlecht, Bildung, Familienstand und Besitz von Land- oder Wohneigentum. Hier könnte man argumentieren, dass aufgrund der Besonderheiten der illegalen Grenzüberquerungen (z.B. gefährliche Flussüberquerungen, generelle Situation von Gewalt und Unsicherheit) Frauen systematisch eine geringere Anzahl an Landeswechseln aufweisen als Männer. Weiterhin ließe sich vermuten, dass Migranten mit einem höheren Bildungsstand weniger Landeswechsel unternehmen, da sie über entsprechendes kulturelles Kapital verfügen, welches ihnen alternative Strategien ermöglichen könnte. Verheiratet zu sein und der Besitz von Land- und Wohneigentum könnten ebenfalls die zu erwartende Anzahl der Landeswechsel reduzieren (vgl. hierzu etwa Massey et al. 1994).

Ein weiteres Merkmalsbündel kann sich aus zeitbezogenen Variablen zusammensetzen. So könnte man annehmen, dass mit zunehmendem Lebensalter und mit zunehmender akkumulierter Lebensarbeitszeit die Anzahl der Landeswechsel steigt. Entsprechend der sogenannten Age-Period-Cohort-Effekte (Mayer / Huinink 1990) beeinflussen neben direkten Alterseffekten auch Perioden- und Kohorteneffekte verlaufsbedingte Ereignisse. Hierzu zählen für die hier relevante Fragestellung etwa das Kalenderjahr (Periodeneffekt) bzw. das Lebensalter (Kohorteneffekt) zum Zeitpunkt des ersten Beschäftigungsverhältnisses insgesamt und das Kalenderjahr, das Lebensalter sowie die bereits kumulierten Erwerbsjahre zum Zeitpunkt des ersten Beschäftigungsverhältnisses in den USA.

Für eine detailliertere Analyse der Erwerbsverläufe wurden Variablen in insgesamt fünf Gruppen zusammengestellt, um die Varianz der Anzahl der Landeswechsel der Migranten als abhängige Variable zu erklären. Vier dieser Variablengruppen bezogen sich auf personenbezogene Variablen (individuelle Eigenschaften der Befragten, ihre Einbettung in Familie und Gemeinde mit $n=648$ gültigen Personenfällen) und eine Gruppe bezog sich auf erwerbsverlaufsbezogene Variablen (Bedingungen für jedes Beschäftigungsverhältnis im Verlauf der 
Migrationsgeschichte der befragten Person mit n=3060 gültigen Beschäftigungsereignissen). Die vier Gruppen der personenbezogenen Variablen waren: (1) Variablen, die sich direkt auf die Person beziehen (Geschlecht, Bildung, Familienstand und Eigentumsbesitz), (2) familienbezogene Variablen (Bildungsniveau des Ehepartners und der Eltern, Migrationsgeschichte des Lebenspartners, der Eltern und der Kinder, Hauptverdiener des Haushaltes), (3) auf die Herkunftsgemeinde in Mexiko bezogene Variablen (sozio-ökonomische Entwicklung, Kommunikations- und Transportinfrastruktur, der Beginn der umfangreicheren Migrationsbewegungen aus der Herkunftsregion) und (4) zeitbezogene Variablen (Alter zum Zeitpunkt der Umfrage, Alter beim ersten Beschäftigungsverhältnis, Zeitpunkt des ersten Beschäftigungsverhältnisses in den USA, Gesamtarbeitszeit in Lebensjahren und Gesamtarbeitszeit in den USA). In der Gruppe der auf den Erwerbsverlauf bezogenen Variablen wurde noch einmal eine Unterscheidung danach vorgenommen, ob das entsprechende Beschäftigungsverhältnis in den USA oder in Mexiko realisiert wurde (bestimmte Fragen wie z.B. die nach Geldrücküberweisungen, nach der ethnischen Zusammensetzung der Arbeitskollegen oder nach der Wohnungssuche im Zusammenhang der jeweiligen Beschäftigung ergaben für Erwerbsereignisse in Mexiko keinen oder einen wesentlich anderen Sinn als für Erwerbsereignisse in den USA).

Die Ergebnisse der personenbezogenen Datenanalyse sind in Tabelle 1 dargestellt. Für die nicht zeitbezogenen personenbezogenen unabhängigen Variablen wurde mit Hilfe des SPSSProgramms eine CATREG-Analyse vorgenommen (welche den Vorteil bietet, unabhängige Variablen unterschiedlicher Skalenniveaus in einem Modell zu integrieren). Bei dieser Analyse wurden die abhängige Variable (Anzahl der Landeswechsel) metrisch und die unabhängigen Variablen entsprechend ihrem Skalenniveau kategorial, ordinal oder numerisch definiert. Für die zeitbezogenen, personenbezogenen, unabhängigen Variablen wurde ein einfaches Regressionsmodell gerechnet. Zunächst zeigt sich, dass die in den vier Gruppen zusammengefassten Variablen (personenbezogene, zeitbezogene, familienbezogene und gemeindebezogene Variablen) jeweils mit einer sehr hohen Wahrscheinlichkeit von mindestens $99 \%$ die Anzahl der Landeswechsel der Befragten beeinflussen. Bei der Betrachtung der Stärke dieser Beeinflussung (Regressionskoeffizienten $\mathrm{R}^{2}$ ) zeigen sich jedoch sehr große Unterschiede.

\section{Tabelle 1: Personenbezogene Variablen zur Erklärung der Landeswechselanzahl}

\begin{tabular}{|c|c|c|c|c|}
\hline Variablengruppe & Unabhängige Variable & Beta* & $\begin{array}{l}\text { Rel.Gewicht für } \\
\text { CATREG }\end{array}$ & $\mathrm{R}^{2}$ \\
\hline \multirow{4}{*}{ 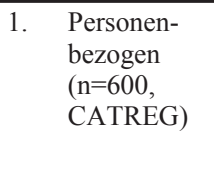 } & Geschlecht & .046 & 6.5 & \multirow{4}{*}{$\begin{array}{l}.041 * * \\
\text { toler. coeff. } \\
>0.97\end{array}$} \\
\hline & Schulniveau & .138 & 52.0 & \\
\hline & Heiratsstatus & .113 & 37.9 & \\
\hline & Landbesitz & .030 & 2.9 & \\
\hline \multirow{5}{*}{$\begin{array}{ll}\text { 2. } & \begin{array}{l}\text { Zeitbezogen } \\
(\mathrm{n}=648,\end{array} \\
& \mathrm{REG})\end{array}$} & Alter zum Interv.zeitpkt & 0.19 & & $0.036^{* *}$ \\
\hline & Kumul. Arbeitszeit ges. & 0.213 & & $0.045^{* *}$ \\
\hline & Kumul. Arbeitszeit USA & -.202 & & $0.041^{* *}$ \\
\hline & Alter beim 1. Jobeinstieg & 0.247 & & $0.061 * *$ \\
\hline & Alter beim 1. Job USA & -0.044 & & n.s. \\
\hline
\end{tabular}




\begin{tabular}{|c|c|c|c|c|}
\hline Variablengruppe & Unabhängige Variable & Beta* & $\begin{array}{l}\text { Rel.Gewicht für } \\
\text { CATREG }\end{array}$ & $\mathrm{R}^{2}$ \\
\hline \multirow[t]{9}{*}{3.} & Schulniveau Vater & -.135 & 62.3 & \multirow{5}{*}{$\begin{array}{l}.035^{* *} \\
\text { toler. coeff. } \\
>0.84\end{array}$} \\
\hline & Schulniveau Mutter & -.058 & 17.7 & \\
\hline & Migration Vater & .044 & 10.0 & \\
\hline & Migration Mutter & .047 & 8.5 & \\
\hline & Migration Geschwister & -.026 & 1.8 & \\
\hline & Schulniveau Partner & -.067 & 24.8 & \multirow{2}{*}{$\begin{array}{l}.027 \\
\text { toler. coef. } \\
>0.93\end{array}$} \\
\hline & Migration Partner & -.134 & 74.0 & \\
\hline & Migration Kinder & -.172 & Y at 0.99 & .030 \\
\hline & Haupt-Haushaltseink. & .125 & $\mathrm{Y}$ at 0.99 & .016 \\
\hline $\begin{array}{l}\text { 4. } \begin{array}{l}\text { Gemeinde- } \\
\text { bezogen } \\
(\mathrm{n}=648,\end{array} \\
\text { CATREG) }\end{array}$ & Herkunftsregion & .204 & 100 & $.041^{* *}$ \\
\hline
\end{tabular}

* Beta $=$ z-transformierter Regressionskoeffizient $* * \mathrm{p}<0,001$

Die personenbezogenen Variablen Geschlecht, Erziehung, Partnerstatus und Landeigentum erklären 4,1 \% der Gesamtzahl der Landeswechsel der befragten Arbeitsmigranten. Es ist bemerkenswert und entspricht nicht den Erwartungen, dass weder die Variable Geschlecht noch die Variable Landeigentum einen sehr starken Einfluss auf die Anzahl der Landeswechsel haben. Ihr relatives Gewicht in dem Multiplen Regressionskoeffizienten von 4,1 beträgt nur $6.5 \%$ bzw. $2.9 \%$. Der ,gender'-Aspekt beeinflusst zwar das spezifische Muster von Erwerbskarrieren (z.B. die Branchenzugehörigkeit und die Aufwärtsmobilitätschancen), aber er erscheint nicht als Korrelationseinfluss in Bezug auf die Anzahl der Landeswechsel. Dies bedeutet nicht, dass damit ein Einfluss der Variable Geschlecht auf die Anzahl von Landeswechseln grundsätzlich ausgeschlossen ist; denkbar wäre etwa, dass in einem frühen Stadium von Migrationsprozessen aus einer bestimmten Herkunftsregion vorwiegend Männer oder aber vorwiegend Frauen migrieren und sich dieser, gender'-Effekt im weiteren Lauf der Zunahme von Migrationsströmen abschwächt. Generell aber steht zu vermuten, dass Männer und Frauen in vergleichbarem Ausma $\beta$ den idealtypischen Gruppen der Einwanderer, Rückkehrwanderer und der Transmigranten angehören (vgl. Herrera Lima 2002; Goldring 2001).

Nach den Untersuchungen von Massey et al. (1998) ist die Variable Landeigentum sehr bedeutsam für die Erklärung der Migrationswahrscheinlichkeit (vgl. auch Díez-Cañedo 1984: $131 \mathrm{f}$ ). Diese Befunde müssen jedoch eventuell auf die Wahrscheinlichkeit, überhaupt zu wandern, begrenzt werden, da sie nicht für die Häufigkeit von Landeswechseln im Erwerbsverlauf zutreffen. Die am stärksten erklärende Variable in dieser Gruppe ist das Bildungsniveau mit einem Anteil von $52 \%$ am (insgesamt nicht sehr starken) Regressionskoeffizienten. Je höher das Bildungsniveau, desto wahrscheinlicher ist eine größere Anzahl von Landeswechseln im Zuge der Arbeitsmigration. Dieser Befund deckt sich mit der Beobachtung von Portes (2003) in Bezug auf transnationale Entrepreneurs. Eine intensivere Analyse zeigt eine erstaunliche Polarisierung im Hinblick auf das Bildungsniveau: Befragte mit einem sehr niedrigen Bildungsniveau (weniger als die sechsjährige Primaria-Schule) und solche mit einem sehr hohen Bildungsniveau (mindestens Abschluss der der Sekundarstufe II vergleichbaren PreparatoriaSchule) wiesen eine signifikant höhere Anzahl an Landeswechseln auf. Bei der Variable ,Partnerstatus' gaben Verheiratete signifikant mehr Landeswechsel an als Alleinstehende, NichtVerheiratete in freien Lebensgemeinschaften und Verwitwete (dies entspricht Befunden bei 
Wiest 1973 und Portes 2003). Familienverbindlichkeiten scheinen die Migrationshäufigkeiten zu erhöhen.

Um Kollinearitätseffekte zu vermeiden, wurden die zeit- und familienbezogenen Variablen in getrennten Untergruppen behandelt. Generell haben außer der Variable, Alter bei Aufnahme der ersten Beschäftigung in den USA' alle zeitbezogenen Variablen einen signifikanten, in seiner Stärke jeweils ähnlich großen Einfluss auf die Anzahl der Landeswechsel wie alle personenbezogenen Variablen zusammengenommen. Von allen zeitbezogenen Variablen beeinflusst das Alter des Befragten zum Interviewzeitpunkt die Anzahl der Landeswechsel mit einem z-transformierten Regressionskoeffizienten von 0,19, was 3,6 \% der gesamten gemessenen signifikanten Beeinflussung der Zahl der Landeswechsel entspricht. Ähnlich korreliert wie zu erwarten - die Anzahl der Landeswechsel mit der kumulierten Beschäftigungsdauer: je älter die Befragten waren, desto eher wiesen sie eine große Anzahl an Landeswechseln auf.

Allerdings war die Beziehung zwischen der Anzahl der Landeswechsel und der kumulierten Beschäftigungszeit in den USA genau umgekehrt: Diejenigen Befragten mit hoher kumulierter Beschäftigungsdauer in den USA gaben tendenziell verhältnismäßig weniger Landeswechsel an. Dies könnte als Hinweis darauf gewertet werden, dass diejenigen mit extrem ausgedehnten Aufenthaltsdauern in den USA dem Idealtypus der Einwanderer nahe kommen. Ein ähnlich negativer (allerdings statistisch weniger signifikanter) Zusammenhang besteht zwischen der Anzahl der Landeswechsel und dem Alter der Befragten zum Zeitpunkt ihres ersten Beschäftigungsverhältnisses in den USA: Je jünger die Befragten bei ihrem ersten US-Beschäftigungsverhältnis, desto häufiger wechseln sie in der Regel zwischen beiden Ländern. Dies könnte ein Indiz für dafür sein, dass Phänomene transnationaler Migration (wie hier die häufigen Landeswechsel) umso gehäufter auftreten, je früher die Arbeitsmigranten mit Erwerbsphasen in den USA beginnen. Im Umkehrschluss könnte man vermuten, dass erst in einem relativ hohen Alter zum ersten Mal in den USA arbeitende Mexikaner von ihrem gesamten Merkmalsset her eher dem Typus der Rückkehrwanderer nahe kommen.

Besonders auffällig ist, dass die familienbezogenen Variablen eine sehr starke Korrelation mit der Anzahl der Landeswechsel aufweisen. Die Summe der vier Korrelationskoeffizienten in dieser dritten Gruppe in Tabelle 1 ist vom Niveau her mehr als doppelt so hoch wie der Korrelationskoeffizient für die vier personenbezogenen Variablen der ersten Gruppe (auch wenn ein direkter zahlenmäßiger Vergleich aus methodischen Gründen nicht möglich ist). Die Schulbildung und Migration des Vaters sowie die Migration des Lebenspartners und der (möglichen) Kinder korrelieren signifikant mit der Anzahl der Landeswechsel (je niedriger die Schulbildung des Vaters und je ausgeprägter USA-Migrationserfahrungen des Vaters, desto häufiger Landeswechsel des Befragten). Wenn der Befragte der Hauptbeiträger zum Haushalteinkommen ist, so erhöht dies ebenfalls statistisch die Zahl seiner Landeswechsel. Diese Ergebnisse decken sich mit den Befunden qualitativer Fallstudien zu den Haushaltsstrategien ländlicher Migrantenhaushalte (Conway / Cohen 2003) und zu den Strategien transnationaler Familien (Bryceson / Vuorela 2002).

Neben den bisher berücksichtigten personenbezogenen Variablen, bei denen jeweils die Gesamtzahl der befragten Arbeitsmigranten $(n=648)$ in Rechnung gestellt wurde, wurden auch die auf die jeweiligen Erwerbsereignisse bezogenen Daten zur Erklärung der Landeswechsel herangezogen. Da jeder Befragte im Durchschnitt 4,7 Beschäftigungsereignisse angab, ist die Zahl der hier zu berücksichtigenden Variablensets wesentlich größer. Die Tabelle 2 enthält wesentliche Ergebnisse der für diesen Fall durchgeführten kategorialen Regressionsanalyse. 
Tabelle 2: Erwerbsverlaufsbezogene Variablen zur Erklärung der Landeswechselanzahl

\begin{tabular}{|c|c|c|c|c|}
\hline Variablengruppe & Unabhängige Variable & Beta* & $\begin{array}{l}\text { Rel. Gewicht } \\
\text { für CATREG }\end{array}$ & $\mathrm{R}^{2}$ \\
\hline \multirow{11}{*}{ Ingsverhältnisse } & Beruf/Tätigkeit & .169 & 23.8 & \multirow{11}{*}{$\begin{array}{l}.142 * * \\
\text { toler. coeff. } \\
>0.79\end{array}$} \\
\hline & Ort des Betriebes & -.091 & 8.0 & \\
\hline & Beschäftigtenanzahl Betrieb & -.056 & 0.5 & \\
\hline & Beziehung zum Arbeitgeber & .097 & 7.4 & \\
\hline & Art des Arbeitsvertrages & .051 & 2.3 & \\
\hline & $\begin{array}{l}\text { Herkunft der (mehrheitl-) Ar- } \\
\text { beitskollegen }\end{array}$ & .096 & 6.2 & \\
\hline & $\begin{array}{l}\text { Informationsquelle für Be- } \\
\text { schäftigung }\end{array}$ & .210 & 35.6 & \\
\hline & $\begin{array}{l}\text { Lohnunterschied zu vorheri- } \\
\text { ger Beschäftig. }\end{array}$ & .028 & 0.8 & \\
\hline & $\begin{array}{l}\text { Wer traf Beschäft.wechsel- } \\
\text { entscheidung }\end{array}$ & .067 & 3.0 & \\
\hline & $\begin{array}{l}\text { Grund für Beschäftigungs- } \\
\text { wechsel }\end{array}$ & -.997 & 8.0 & \\
\hline & Wöchentliche Arbeitszeit & .071 & 4.4 & \\
\hline \multirow{5}{*}{$\begin{array}{ll}5 \mathrm{~b} . & \begin{array}{l}\text { Nur Beschäfti- } \\
\text { gungsverhältnisse }\end{array} \\
\text { in USA } \\
\text { ( }=905, \\
\text { CATREG) }\end{array}$} & $\begin{array}{l}\text { Ethnische Zugehörigkeit Ar- } \\
\text { beitgeber }\end{array}$ & .171 & 22.3 & \multirow{5}{*}{$\begin{array}{l}.144 * * \\
\text { toler. coeff. } \\
>0.98\end{array}$} \\
\hline & Legaler Aufenthaltsstatus & .085 & 3.6 & \\
\hline & Hilfe bei Unterkunftssuche & .047 & 1.2 & \\
\hline & $\begin{array}{l}\text { Wichtigster Geldüberwei- } \\
\text { sungsempfänger }\end{array}$ & .268 & 54.5 & \\
\hline & $\begin{array}{l}\text { Zweitwichtigster Geld- } \\
\text { überw.empfänger }\end{array}$ & .159 & 18.4 & \\
\hline
\end{tabular}

$*$ Beta $=$ z-transformierter Regressionskoeffizient

$* * \mathrm{p}<0,001$

Bei der Analyse der auf die Arbeit bezogenen Variablen (siehe Tabelle 2) liegt die Anzahl der gültigen Fälle bei über 1000 für alle Beschäftigungsverhältnisse und bei einer Anzahl von $\mathrm{n}=905$, die sich nur auf Beschäftigungsverhältnisse in den USA beziehen (siehe den Abschnitt 5 b der Tabelle 2). Jeder der beiden Gruppenabschnitte der Tabelle 2 erklärt für sich etwas mehr als $14 \%$ der Variation in der Anzahl der Landeswechsel der Befragten. Betrachtet man die Z-transformierten Koeffizienten (Beta) für jede Variable und ihre relative Gewichtung im gesamten Multiplen Regressionskoeffizienten für alle Beschäftigungsverhältnisse (5 a), so erweisen sich die folgenden zwei Variablen als am wichtigsten für die Erklärung der Anzahl der Landeswechsel: Beruf (manuelle Arbeit in der Industrie senkt die Wahrscheinlichkeit vieler Landeswechsel; im Gegensatz dazu erhöht eine Arbeit im Restaurantgewerbe, im Hotelgewerbe oder im Handel die Wahrscheinlichkeit von vielen Landeswechseln) und Informationsquelle zur Arbeitsbeschaffung (die Anzahl der Landeswechsel nimmt in dem Ausmaß zu, wie die Informationsbeschaffung für Beschäftigungen weniger durch Familienangehörige und Verwandte erfolgt).

Der Fragebogen enthielt einige Variablen, die sich speziell auf die Arbeitssituation in den USA und nicht auf Beschäftigungsverhältnisse in Mexiko beziehen (wie zum Beispiel Auf- 
enthaltstatus, siehe Abschnitt $5 \mathrm{~b}$ der Tabelle 2). Diese Variablen tragen ebenfalls zu etwa $14 \%$ zur Erklärung aller Landeswechsel bei. Der Anteil an Arbeitgebern in den USA, die aus der gleichen Herkunftsgemeinde wie die Befragten stammen, verringert sich mit der Anzahl an erfolgten Landeswechseln, und zugleich erhöht sich mit der Anzahl der Landeswechsel die Wahrscheinlichkeit, dass der Arbeitgeber kein Lateinamerikaner ist. Eine Hilfestellung durch Familienangehörige oder Freunde beim Grenzübergang nimmt ebenso mit der Anzahl der Landeswechsel ab. Mit einer wachsenden Anzahl von Bewegungen zwischen Mexiko und den USA ändern sich die Adressaten der Geldrücküberweisungen: Zuerst richten sich diese an die Eltern, später an die Lebenspartner und Kinder. Diese Variablen (wichtigste und zweitwichtigste Adressaten für remittances) machen zusammen etwa drei Viertel der Varianzerklärung in dieser Gruppe der US-Beschäftigungsverhältnisse aus. Dies ist ein klarer Hinweise auf die große Bedeutung von Familiennetzwerken für die Erklärung der Häufigkeit der Landeswechsel. In diesem Zusammenhang ist überraschend, dass der Aufenthaltsstatus nicht signifikant mit der Anzahl der Landeswechsel korreliert und nur ein sehr geringes Gewicht $(3,6 \%)$ hat.

Insgesamt erlaubt die Datenanalyse ein differenziertes Bild der Faktoren zu zeichnen, die einen Einfluss auf die Häufigkeit der Landeswechsel der befragten mexikanischen Arbeitsmigranten haben. Es zeigt sich zunächst, dass nicht alle Befragten den idealtypischen Mustern von Auswanderern/Einwanderern und von Rückkehrwanderern entsprechen. Eine beträchtliche Anzahl von Migranten pendelt zwischen dem Großraum New York und der Puebla-Region hin und her und verbleibt dabei jeweils nicht nur saisonal, sondern für einige Jahre in den USA. Es handelt sich hierbei nicht einfach um , gescheiterte Einwanderer', da sie in aller Regel ihre Beschäftigungsverhältnisse in den USA aus ,freiwilligen' Gründen aufgeben, und zwar fast ausschließlich aus familiären Gründen. Die engen Familiennetzwerke sind auch entscheidend bei der Organisation zumindest der ersten Migrationsaufenthalte in den USA (Informationsbeschaffung, Finanzierung und Organisation der Migration; Arbeitssuche, Unterkunft, Geldrücküberweisungen). Familien-, zeit- und arbeitsbezogene Variablen erklären die Anzahl (und auch die Dauer) ${ }^{7}$ der Aufenthalte in einem der Länder. Von allen auf die Beschäftigungsverhältnisse bezogenen Variablen erklären diejenigen am meisten in Bezug auf die Varianz der Landeswechsel, welche sich auf die Familien der Befragten beziehen oder familiäre Aspekte beinhalten (wie z.B. die familiären Gründe für die Beendigung eines Beschäftigungsverhältnisses und die Geldrücküberweisungen).

\section{Schlussfolgerung}

Obwohl viele der analysierten Erwerbsverläufe mexikanischer Arbeitsmigranten bekannte Muster aufweisen, zeigen sich bei einem nicht unerheblichen Anteil von Migranten neue Formen von Migration: Sie richten ihre Biographie und Lebensstrategie weder exklusiv an der Herkunftsregion noch ausschließlich an der Ankunftsregion aus. Ihre häufigen Wechsel von Wohnort und Beschäftigung und auch ihre subjektiven Erwerbsstrategien und -orientierungen spannen sich zwischen Mexiko und den USA auf. Der zeitliche Horizont ihrer Lebensplanung ist nicht eindeutig fixiert oder für einen unbegrenzten Zukunftshorizont festgelegt. Vielmehr planen sie ihr ,Lebensprojekt' eher sequentiell und iterativ in überschaubaren Zeitabschnitten von mehreren Jahren (etwa nach dem generellen Muster: ,nur Gott kennt mein Schicksal, ich plane jetzt erst mal die nächsten zwei oder drei Jahre und dann sehen wir weiter').

Ein sehr dichtes transnationales Netzwerk von Verwandtschafts- und Freundschaftsbeziehungen verdichtet sich dabei zu einem Sozialraum, der ihnen Sicherheit und Orientierung verleiht. Dieser transnationale Sozialraum ermöglicht ihnen, relativ problem- und risikolos

7 Ähnliche Berechnungen wie die hier vorgestellten wurden neben der abhängigen Variable der Anzahl der Landeswechsel auch für die Variable der Dauer der jeweiligen Beschäftigungsverhältnisse in den USA durchgeführt. Die Ergebnisse decken sich weitgehend mit den hier vorgestellten Befunden. 
zwischen beiden Ländern - physisch und mental - zu wechseln. Auf der Basis dieser sozialräumlichen Beziehungen sowie der damit gegebenen Orientierungen und Sicherheiten finden sie z.B. Arbeit in New York City; aufgrund dieser sozialräumlichen Beziehungen sowie der damit verbundenen Loyalitäts- und Reziprozitätsbeziehungen kehren sie z.B. für einige Zeit nach Mexiko zurück. Die Analyse in Abschnitt 4 verwies auf die herausgehobene und signifikante Bedeutung familienbezogener Variablen zur Erklärung der Anzahl von Landeswechseln.

Der Anteil der dem Idealtypus von Transmigranten nahe kommenden mexikanischen Arbeitsmigranten beträgt je nach Enge oder Weite der Definition ein Sechstel bis ein Viertel der Befragten. Auswanderer/Einwanderer, Rückkehrwanderer und Transmigranten stellen theoretisch-analytische Idealtypen dar, deren ,Erklärungsmächtigkeit' empirisch überprüfbar ist. Hinweise auf die Erklärungskraft und Nützlichkeit des Idealtypus Transmigrant können schon in der Geschichte der internationalen Migration gefunden werden (Smith 2001). Aber zu Beginn des 21. Jahrhunderts erscheint dieser Idealtypus unverzichtbar, um die soziale Realität und Dynamik internationaler Wanderungsprozesse zu verstehen und zu erklären. Die klassischen Migrantenkategorien (Einwanderer, Rückkehrwanderer und Pendel- oder Diaspora-Migrant) reichen nicht mehr aus, um der migratorischen Realität von Menschen gerecht zu werden. Der Idealtypus des Transmigranten erscheint als eine sinnvolle weitere Kategorie, um Wanderungsmuster zu erfassen.

Transmigranten und Transmigration unterminieren empirisch und theoretisch das Konzept von homogenen nationalen Containergesellschaften, die in territorial voneinander abgeschlossene Nationalstaaten eingebettet sind. Durch transnationale Sozialräume, wie sie im Rahmen von Transmigration entstehen und gefestigt werden können, wird das Konzept nationalstaatlicher Souveränität und nationalgesellschaftlicher Homogenität relativiert. Die mächtigen Migrationsströme von Mexiko in die USA unterminieren in vielfacher Hinsicht (und ähnlich wie auch andere Prozesse der wirtschaftlichen, politischen, kulturellen und sozialen Internationalisierung) den mexikanischen Nationalstaat und die ,mexicanidad' der dortigen Nationalgesellschaft. Umgekehrt erklärten mexikanische Staatspräsidenten seit den 1990er Jahren wiederholt, dass die Grenzen der mexikanischen Nation durchaus nicht an den Territorialgrenzen des mexikanischen Staates endeten, sondern dass Millionen von mexikanischen Landsleuten in anderen Ländern, vor allem den USA lebten. Diese seien aktiv in die mexikanische Nation einzubeziehen.

Zusammengefasst erweist sich der Typus des Transmigranten und das Transnationalisierungskonzept insgesamt als eine fruchtbare Erweiterung und Differenzierung in der allgemeinen Forschung und Diskussion über internationale Migration und über Globalisierung insgesamt. Die Globalisierungsdebatten der letzten zwei Jahrzehnte haben sich vor allem auf die emergierenden ökonomischen, kulturellen und politischen Aspekte der Dynamik und Struktur von Unternehmen, öffentlichen und non-profit Organisationen konzentriert. Der Fokus der transnationalen Migration richtet sich dagegen vor allem auf Formen der ,Transnationalisierung von Unten' (Smith / Guarnizo 1999; Ong / Nonini 1997), welche durch soziale Praktiken, Symbolsysteme und Artefakte von Millionen von Akteuren vorangetrieben wird. Auch für die europäische Migrationswirklichkeit ergeben sich wichtige Anknüpfungspunkte, wie neuere Forschungen zeigen (Faist 2000 b; Hillmann 2000; Mau 2007; Pries 2005 b; Sandu 2005). Forschungen zur transnationalen Migration sollten die bewährten klassischen Untersuchungsansätze nicht ersetzen, sondern helfen, diese weiterzuentwickeln und auszudifferenzieren. Nach vielen konzeptionellen Debatten bleibt hier vor allem noch viel empirische Arbeit zu tun. 


\section{Literatur}

Basch, L. / Glick Schiller, N. / Szanton Blanc, C. (1997): Nations Unbound. Transnational Projects, Postcolonial Predicaments, and Deterritorialized Nation-States, Amsterdam.

Bryceson, D. / Vuorela, U. (2002): The Transnational Family: New European Frontiers and Global Networks, Oxford.

Bustamante, J.A. / Martínez, G.G. (1979): Undocumented Migration from Mexico: Beyond Borders but with Systems, in: Journal of International Affairs 33, S. 265-284.

Chimal, C. (1990): Movimiento perpetuo. Mixtecos en California, in: México Indígena 4.

Cederström, T.N. (1993): The Impacts of Migrant Remittances on the Peasant Economy of Four Communities of the Mixteca Baja Region of Puebla, Mexico.

Cohen, J.H. (2001): Transnational Migration in Rural Oaxaca, Mexico: Dependency, Development, and the Household, in: American Anthropologist 103, S. 954-967.

Conway, D. / Cohen, J. (2003): Local Dynamics in Multi-local, Transnational Spaces of Rural Mexico: Oaxacan Experiences, in: International Journal of Population Geography 9, S. 141-161.

Cortés Sánchez, S. (1995 a): La Mixteca, tierra de emigrados, in: Perfil de La Jornada de Oriente, 08.03.1995.

Cortés Sánchez, S. (1995 b): Los migrantes de la Mixteca poblana, in: La Jornada de Oriente, 29.11.1995.

Cortés Sánchez, S. (1996): El retorno de los chinantlecos, in: La Jornada de Oriente, 31.01.1996.

Corona Vázquez, R. (1993): Migración permanente interestatal e internacional, 1950-1990, in: Comercio Exterior, S. 750-762.

Durand, J. / Massey, D.S. (1992): Mexican Migration to the USA, in: Latin American Research Review 27(2), S. 3-42.

Díez-Canedo Ruiz, J. (1984): La migración indocumentada de México a los Estados Unidos. Un nuevo enfoque, México.

Espinosa, K. / Massey, D. (1997): Undocumented Migration and the Quantity and Quality of Social Capital, in: L. Pries (Hrsg.), Transnationale Migration. Sonderband 12 der Zeitschrift Soziale Welt, BadenBaden, S. 141-162.

Faist, T. (2000 a): The Volume and Dynamics of International Migration and Transnational Social Spaces, Oxford.

Faist, T. (2000 b): Transnationalization in international migration: implications for the study of citizenship and culture, in: Ethnic \& Racial Studies 23, S. 189-222.

Faulstich Orellana, M. / Thorne, B. / Chee, A. / Lam, W.S.E. (2001): Transnational Childhoods: The Participation of Children in Processes of Family Migration, in: Social Problems 48, S. 572-591.

Goldring, L. (1996): Blurring the Border: Transnational Community and Social Transformation in Mexico-U.S. Migration, in: D. Chekki (Hrsg.), Research in Community Sociology, Band 6, Stamford / London.

Goldring, L. (1997): Power and Status in Transnational Social Spaces, in: L. Pries (Hrsg.), Transnationale Migration. Sonderband 12 der Zeitschrift Soziale Welt, Baden-Baden, S. 179-196.

Goldring, L. (2001): Dissagregating Transnational Social Spaces: Gender, Place and Citizenship in Mexico-U.S. Transnational Spaces, in: L. Pries (Hrsg.), New Transnational Social Spaces. International Migration and Transnational Companies. London, S. 59-76.

González, S. / Ruiz, O. / Velasco, L. / Woo, O. (1995): Mujeres. Migración y maquila en la frontera norte, México.

Guarnizo, L.E. (2003): The Economics of Transnational Living, in: International Migration Review 37, S. 666-699. 
Heer, D.M. (1993): Los mexicanos indocumentados en los Estados Unidos, México.

Hernández, A. (1990): Mixtecos en Baja California. Destino San Quintín, in: México Indígena 11.

Herrera Lima, F. (2001): Transnational Families: Institutions of Transnational Social Space, in: L. Pries (Hrsg.), New Transnational Social Spaces. International Migration and Transnational Companies, London, S. 77-93.

Herrera Lima, F. (2002): Trayectorias y Biografias Laborales en la Migracion de Puebla y tlaxcala y la Zona Metropolitana de la Ciudad de Nueva York. PhD Thesis, Department of Anthropology, México.

Herrera Lima, F. / Macías Reyes, S. (1998): Migración de la Mixteca Poblana a Nueva York: Espacio social transnacional, in: S. Macías Gamboa / F. Herrera Lima (Hrsg.), Migración laboral internacional, Puebla, S. 107-163.

Hillmann, F. (2000): Von internationalen Wanderungen zu transnationalen Migrationsnetzwerken? Der neue europäische Wanderungsraum, in: Bach, Maurizio (Hg.): Die Europäisierung nationaler Gesellschaften. (Sonderband der Kölner Zeitschrift für Soziologie und Sozialpsychologie, Jahrgang 40, 2002), S. 363-385.

Kearney, M. / Nagengast, C. (1989): Anthropological Perspectives on Transnational Communities in Rural California, Davis CA: California Institute for rural Studies, Working Group on Farm Labor and Rural Poverty (Working Paper No. 3).

López Angel, G. / Cederström, T.N. (1990): Moradores en el purgatorio: el regreso periódico de los migrantes como una forma de peregrinación, en: INAH/CNCA (Hrsg.), Izúcar de Matamoros.

López Castro, G. (1986): La casa dividida. Un estudio de caso sobre la migración a Estados Unidos en un pueblo michoacano, Zamora.

López Castro, G. (1988): Migración en el Occidente de México, Zamora.

Lucassen, L. (2006): Is Transnationalism Compatible with Assimilation? Examples from Western Europe since 1850, in: IMIs-Beiträge 29, S. 15-35.

Massey, D.S. / Alarcón, R. / Durand, J. / González, H. (1991): Los ausentes. El proceso social de la migración en el occidente de México, México.

Massey, D. / Arango, J. / Hugo, G. / Kouaouci, A. / Pellegrino, A. / Taylor, J.E. (1994): An Evaluation of International Migration Theory: The North American Case, in: Population and Development Review 20, S. 699-751.

Massey, D.S. / Arango, J. / Hugo, G. / Kouaouci, A. / Pellegrino, A. / Taylor, E.S. (1998): Worlds in Motion. Understanding International Migration at the End of the Millennium, Oxford.

Mau, S. (2007): Transnationale Vergesellschaftung. Die Entgrenzung sozialer Lebenswelten. Frankfurt / M.

Mayer, K.U. / Huinink, J. (1990): Age, period, and cohort in the study of the life course: A comparison of classical A-R-C-analysis with event history analysis or Farewell to Lexis?, in: D. Magnusson / L.R. Bergman (Hrsg.), Data Qualitiy in Longitudinal Research. Cambridge, S. 211-232.

Motta Sánchez, J.A. (1990): 'De Piaxtla, Pue. to New York' noticias sobre algunas consecuencias del trabajo migratorio internacional en una localidad rural de la mixteca poblana, in: INAH/CNCA (Hrsg.), México, S. 119-124.

Mummert, G. (1990): Población y Trabajo en contextos regionales, Zamora.

Ong, A. / Nonini, D. (1997): Ungrounded Empires. The Cultural Politics of Modern Chinese Transnationalism. London / New York.

Palloni, A. / Massey, D.S. / Ceballos, M. / Espinosa, K. / Spittel, M. (2001): Social Capital and International Migration: A Test Using Information on Family Networks, in: American Journal of Sociology 106, S. $1262-1298$.

Portes, A. (1996): Transnational Communities: Their Emergence and Significance in the Contemporary World System, in: W.A. Smith / R.S. Korzeniewicz (Hrsg.), Latin America in the World Economy. Westport: Greenwood Press, S. 151-168. 
Portes, A. (2003): Conclusion: theoretical convergencies and empirical evidence in the study of immigrant transnationalism, in: International Migration Review 37, S. 874-892.

Portes, A. / Guarnizo, L.E. / Landolt, P. (1999): Introduction: Pitfalls and promise of an emergent research field, in: Ethnic and Racial Studies 22, S. 217-237.

Preibisch, K.L. (1996): Neoliberalismo y migración en el campo mexicano: bosquejo de dos comunidades poblanas, in: Economía Internacional 52, S. 73-79.

Pries, L. (1996): Transnationale Soziale Räume. Theoretisch-empirische Skizze am Beispiel der Arbeitswanderungen Mexiko-USA, in: Zeitschrift für Soziologie 25, S. 437-453.

Pries, L. (1998): 'Transmigranten' als ein Typ von Arbeitswanderern in pluri-lokalen sozialen Räumen. Das Beispiel der Arbeitswanderungen zwischen Puebla/Mexiko und New York, in: Soziale Welt 49, S. $135-150$.

Pries, L. (2005 a): Configurations of Geographic and Societal Spaces: A socio-logical proposal between 'methodological nationalism' and the 'spaces of flows', in: Global Networks 5, S. 167-190.

Pries, L. (2005 b): Zwischen den Welten und amtlichen Zuschreibungen. Neue Formen und Herausforderungen der Arbeitsmigration im 21. Jahrhundert, Essen.

Sandu, D. (2005): Emerging Transnational Migration from Romanian Villages, in: Current Sociology 53, S. $555-582$.

Smith, M.P. / Guarnizo, L.E. (1999): Transnationalism from below. Comparative Urban \& Community Research, Band 6. New Brunswick / London.

Smith, R. (1997): Reflections on Migration, the State and the Construction, Durability and Newness of Transnational Life, in: L. Pries (Hrsg.), Transnationale Migration. Sonderband 12 der Sozialen Welt. Baden-Baden, S. 197-220.

Smith, R. (2001): Comparing Local Level Swedish And Mexican Transnational Life. An Essay In Historical Retrieval, in: L. Pries, L. (Hrsg.), New Transnational Social Spaces. International Migration and Transnational Companies. London, S. 37-58.

Smith, R. (2005): Mexican New York: Transnational Worlds of New Immigrants, Los Angeles.

Valdéz, L.M. (1994): Migación laboral de mexicanos a Nueva York, in: Instituto Matías Romero de Estudios Diplomáticos, La migración laboral mexicana a Estados Unidos: una perspectiva desde México, Mexico, S. 207-213.

Valdéz, L.M. (1996): La Gran Mannhatitlán, in: Nexos 7, S. 34-42.

Velasco Ortiz, L. (1995): Entre el jornal y el terruño: los migrantes mixtecos en la Frontera Noroeste de México, in: Nueva Antropología 47, S. 113-129.

Weiß, A. (2005): The Transnationalization of Social Inequality: Conceptualizing Social Positions on a World Scale, in: Current Sociology 53, S. 707-728.

Wiest, R.E. (1973): Wage-Labor Migration and the Household in a Mexican Town, in: Journal of Anthropological Research 29, S. 180-209.

Prof. Dr. Ludger Pries Ruhr-Universität-Bochum

Fakultät für Sozialwissenschaft

Universitätsstr. 150 44780 Bochum ludger.pries@ruhr-uni-bochum.de 